\title{
Lossless Hybrid Coding technique based on Quasi Fractal \& Oscillation Concept Method for Medical Image Compression
}

\author{
Satyawati Magar a ${ }^{\text {, }}$, Bhavani Sridharan ${ }^{\text {a }}$ \\ a Department of E \& TC, D.V.V.P College of Engineering, Ahmednagar, Maharashtra, India. \\ b Department of ECE, Sri Shakthi Institute of Engineering \& Technology Coimbatore, Tamil Nadu, \\ India. \\ * Corresponding Author: magarss_123@rediffmail.com
}

Received : 20"th March 2020, Accepted : $08^{\text {th }}$ May 2020

Abstract: The Image compression is the most important entity in various fields. Image compression plays vital role in many applications. Out of which biomedical is one of the challenging applications. In medical research, everyday there is fast development and advancement. Medical researchers are thinking about digital storage of data hence medical image compression has a crucial role in hospitals. Here Morphological filter \& adaptive threshold are used for refinement and used Quasi Fractal \& Oscillation concept for developing new hybrid algorithm. Oscillation concept is lossy image compression technique hence applied on NonROI. Quasi fractal is lossless image compression technique applied on ROI. The experimental results shows that better CR with acceptable PSNR has been achieved using hybrid technique based on Morphological band pass filter and Adaptive thresholding for ROI. Here, innovative hybrid technique gives the CR 24.61 which improves a lot than hybrid method using BTCSPIHT is 5.65. Especially PSNR is also retained and bit improved i.e. 33.51. This hybrid technique gives better quality of an image.

Keywords: Quasi Fractal, Morphological Filter, Adaptive Threshold, CR, MSE, PSNR, Oscillation Concept, BTC-SPIHT.

\section{Introduction}

Image compression plays vital role in many applications. Out of which biomedical is one of the challenging application. In medical research, everyday there is fast development and advancement. Medical researchers are thinking about digital storage of data hence medical image compression has a crucial role in hospitals. Everyday high amount of medical images are captured in the medical laboratories. Due to this high amount of data, there is big question to store it in less space. Storing the data in less space becomes challenging to practitioners. 
Compression algorithms are developed by the researchers but compression of an image with high CR by keeping excellent image quality is a big challenge. Moreover to avoid diagnostic errors and reduce transmission time is also challenging. Image compression has immense importance as time consumption is very high to process out the original biomedical image. By using image compression algorithms we can compress different biomedical images. To reduce processing time required to retrieve target components from biomedical images, compression of image is necessary. In order to compress images different techniques are used i.e. lossy and lossless. To minimize the file size for storage PACS are used, which helps to keep information maintained. Compression algorithms in medical imaging has become challenging. For image compression many algorithms are used for achieving better CR \& PSNR. In the field of medical there is a challenge to store more data hence image compression is one of the better solutions. Image compression is the scientific art of reducing size which is required for representing an image. These are useful \& successful technologies in DIP field. Daily incalculable number of images is compressed \& decompressed. Many images from web pages \& high resolution cameras are also compressed. Especially it is used to get best accuracy of an image with a high CR. This research aims to improve the CR by keeping good quality of the image. Now a days various image compression techniques are used by researchers. DCT, DFT, DWT \& Fractal are most commonly used existing image compression techniques. In order to achieve better results than these existing techniques we have implemented oscillation concept method. For compression, we can use MRI, CT \& PET image. Both MRI and CT scan are used for detailing of brain images. MRI or CT images are used for Image compression which is effectively used for analysis of brain images. Here we have selected brain image for research. MRI is most effective in brain images hence we have selected MRI brain image for research.

\section{Hybrid Coding Technique}

\subsection{Quasi Fractal \& Oscillation Concept Method}

\subsubsection{Quasi Fractal}

It is new method to code \& decode images. It is simple method to regenerate images without data loss. Fractal based coding algorithms are standard fractal coding, Quasi lossless fractal coding \& Improved Quasi lossless fractal coding. These algorithms are evaluated by checking their ability of compress MRI based CR, PSNR and encoding time. These new lossless fractal methods perform better than existing fractal coding.

This assures applicability of lossless fractal image compression in medical field. Application of fractal compression to medical images would allow much higher CR with good picture quality. Quasi lossless fractal coding has been used for development of hybrid technique $[1,2]$. 


\subsubsection{Oscillation Concept}

Oscillation concept is new approach in image compression. This method explains theory of oscillations in images. Each and every image having vibrations and variations in it. These variations are known as oscillations. These variations are mainly in the pixel of an image. These variations are produced with respect to $\mathrm{x} \& \mathrm{y}$ axis. For compression of an image these oscillations are used. This method introduced for improving CR.

There is variation in grey scale intensities, these are oscillations in an image. This concept is utilized to find out the variations in biomedical images, appropriate oscillations are considered for image compression.

By repeating the process we can obtain the Principal part from image. It is continued till better quality of Principal component is obtained. Here good quality is obtained by extracting PC. It is explained as continuous signal just for understanding purpose.[14]

\subsection{BTC-SPIHT}

Firstly hybrid algorithm is implemented using BTC \& SPIHT. BTC is lossy compression technique and SPIHT is lossless. This hybrid algorithm is used after enhancement.

\subsubsection{Block Truncation Coding (BTC):}

This technique is used for digitized gray scale images which is very fast and simple lossy Image Compression technique. For compressing digital gray level images BTC uses moment preserving quantization method. Even though this method retains the visual quality of the reconstructed image with high data CR and PSNR

\subsubsection{Set Partitioning in Hierarchical Trees (Spiht):}

This is powerful wavelet-based image compression method called SPIHT. The original image is passed through DWT block which outputs DWT coefficients of the original image.

Then DWT coefficients are passed through SPIHT encoder which encodes the output and gives data bit stream manner. This bit stream send through SPIHT decoder and passed through IDWT block, which gives original (reconstructed) image back.

\section{Methodology}

Key factors of innovative hybrid method are morphological filter \& adaptive threshold. This methodology is totally based on multilevel operation. By using this methodology we can achieve required ROI.

\subsubsection{Hybrid method flow diagram}

Hybrid algorithm is developed using Lossy and Lossless image compression techniques. Here for Non-ROI region lossy image compression technique is used and for ROI Lossless image compression technique is used. 
For Lossy compression oscillation concept and for lossless image compression Quasi fractal technique is used. This technique is developed for achieving better results. Hybrid techniques for compression of an image of brain use following steps as shown in figure 1. [5-11]

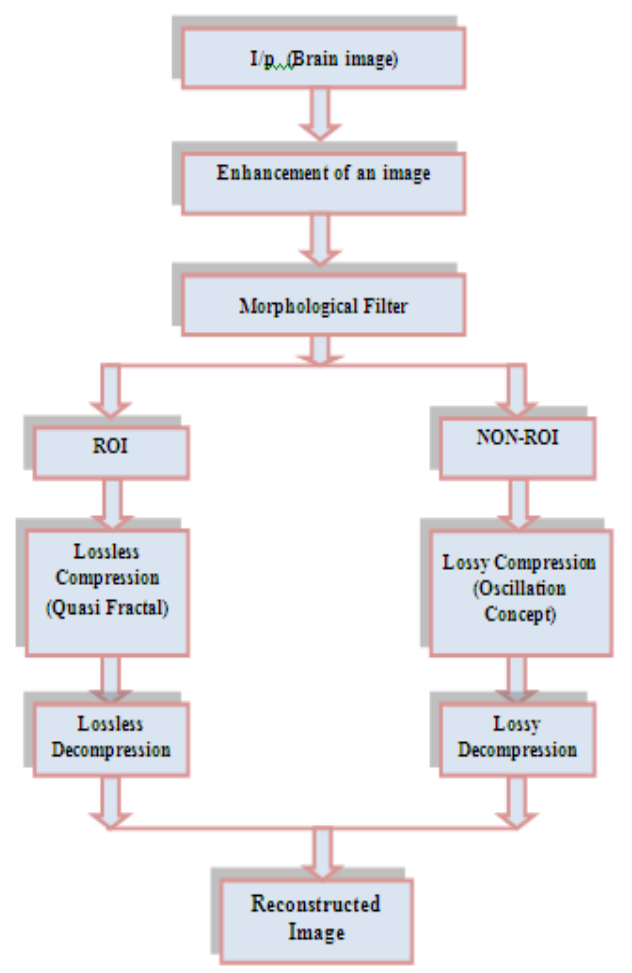

Fig.1 Flow Diagram of Hybrid Technique

\subsubsection{Morphological Filter}

It can be defined as the multilevel open operations. These multilevel open operations are just like a LPF, it eliminates the structures of the image which are smaller than that. A morphological BPF is the difference between two multilevel open operations with different structuring elements. For detecting micro-calcifications Morphological MBF is introduced, which is implemented by opening the original image two times with two different structure elements respectively. Also subtracting one opened image using another one. Due to this microcalcifications tend to appear, MBF's are tuned for the detection task, and binary image containing ROI are obtained. Multilevel morphological operations used concept of umbra (U [ ]) and top (T [ ]) surface. [3,4]

\subsubsection{Adaptive Threshold:}

In image processing thresholding a greyscale image with a fixed value to get a binary image is mostly used operation. Neighbouring pixel intensities are important for deciding the threshold value at each pixel location. Adaptive thresholding is used for partitioning the original 
image into certain sub images and utilize global thresholding techniques for each sub image $[12,13]$.

\subsection{Algorithm for Quasi Fractal \& Oscillation Concept Method}

Step 1. Read Input Image

Step 2. Convert RGB to Gray image

Step 3. Denoise an image

Step 4. Resize image into 256 * 256

Step 5. Filtration by using Morphological Filters

Step 6. Apply Adaptive thresholding

Step 7. Find current ROI

Step 8. Repeat procedure by tuning morphological Filter for Refinement.

Step 9. Find Final ROI

Step 10. Find ROI

Step 11.Apply Lossless Compression Technique over final ROI.

Step 12.Apply Lossy Compression Technique on NonROI.

Step 13.Combine O/P images of Step 11\& Step 12.

Step 14.Show reconstructed compressed image.

\section{Result and Discussion}

\section{Result}

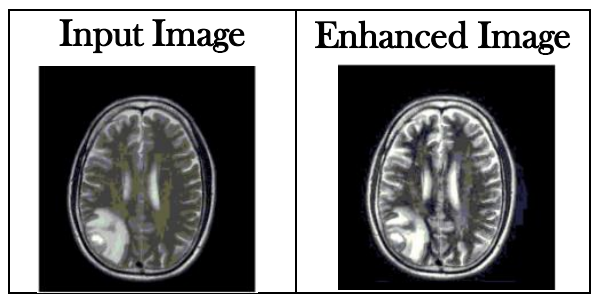


Vol. 2 Iss. 1 Year 2020 Satyawati Magar and Bhavani Sridharan /2020

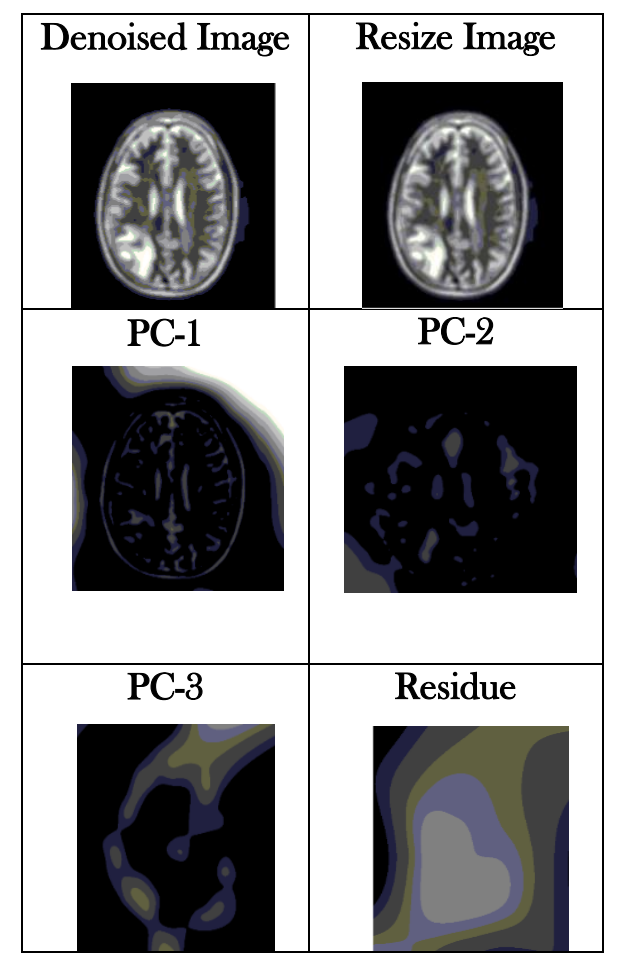

Fig.2 Result snaps of Hybrid coding algorithm using Oscillation Concept \& Quasi Fractal Table.1 Comparative analysis of statistical parameters CR, PSNR,MSE \& MSSIM

\begin{tabular}{|l|l|l|l|l|l|}
\hline Sr. No & Technique used for Image Compression & \multicolumn{4}{|l|}{ Parameters } \\
\cline { 3 - 6 } & & CR & PSNR & MSE & MSSIM \\
\hline 1 & $\begin{array}{l}\text { Hybrid Coding using } \\
\text { Oscillation Concept \& Quasi Fractal }\end{array}$ & 24.61 & 33.51 & 28.96 & 0.58 \\
\hline 2 & $\begin{array}{l}\text { Hybrid Coding using } \\
\text { BTC-SPIHT }\end{array}$ & 5.65 & 33.01 & 32.51 & 0.55 \\
\hline
\end{tabular}


Vol. 2 Iss. 1 Year 2020 Satyawati Magar and Bhavani Sridharan /2020

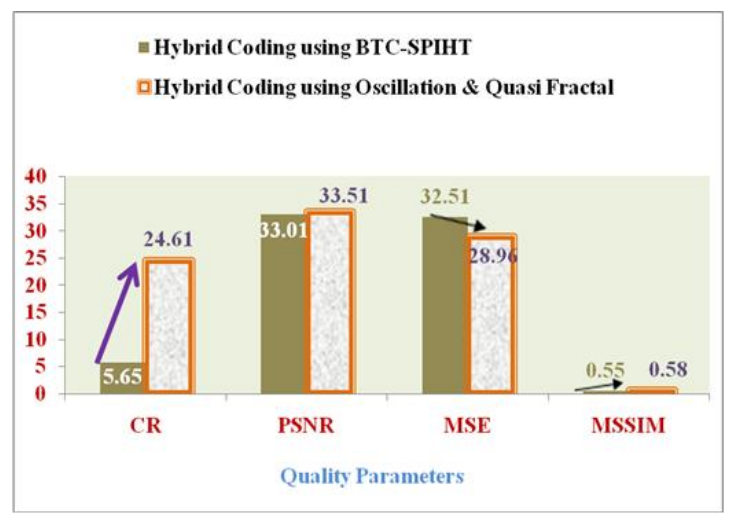

Fig.3 Comparative Analysis Between two hybrid methods

Table 2 Results of Hybrid Method for various brain images

\begin{tabular}{|l|l|l|l|l|}
\hline MRI Images/ Parameters & CR & PSNR & MSE & SSIM \\
\hline IMG-1 & 24.61 & 34.15 & 24.96 & 0.58 \\
\hline IMG-2 & 22.41 & 33.0985 & 31.8586 & 0.48 \\
\hline IMG-3 & 20.69 & 41.4213 & 4.6876 & 0.51 \\
\hline IMG-4 & 20.3 & 34.4859 & 23.1465 & 0.5 \\
\hline IMG-5 & 21.78 & 37.7 & 11.04 & 0.596 \\
\hline IMG-6 & 20.17 & 41.6582 & 4.4387 & 0.6 \\
\hline IMG-7 & 23.09 & 34.966 & 20.7242 & 0.527 \\
\hline IMG-8 & 28.96 & 37.7 & 11.04 & 0.6 \\
\hline IMG-9 & 23.96 & 32.93 & 33.05 & 0.74 \\
\hline IMG-10 & 23.59 & 33.42 & 29.5225 & 0.527 \\
\hline AVG & 22.956 & 36.15299 & $\mathbf{1 9 . 4 4 6 8 1}$ & 0.566 \\
\hline
\end{tabular}


Vol. 2 Iss. 1 Year 2020 Satyawati Magar and Bhavani Sridharan /2020

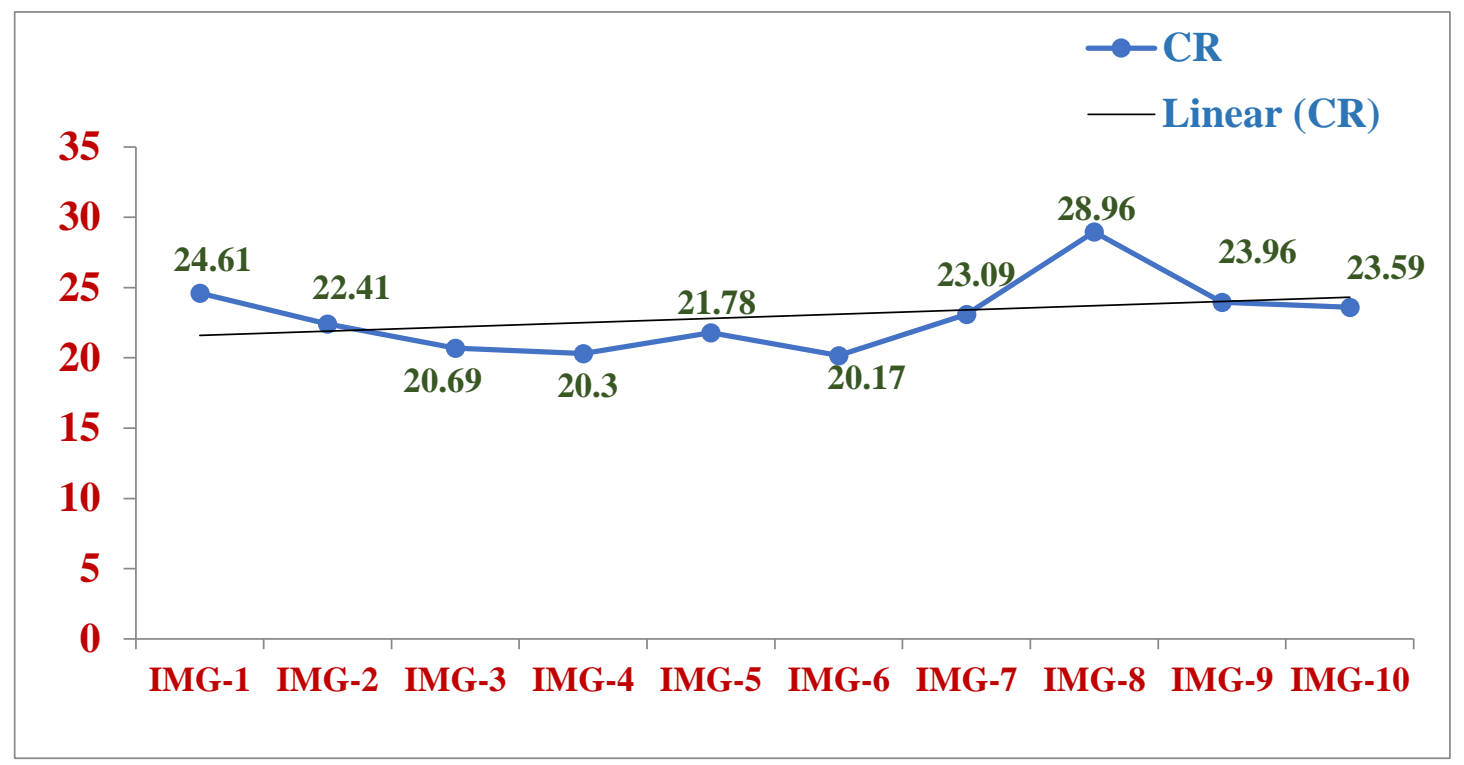

Fig.4 Analysis of CR for Various Brain Images using Hybrid Techniques

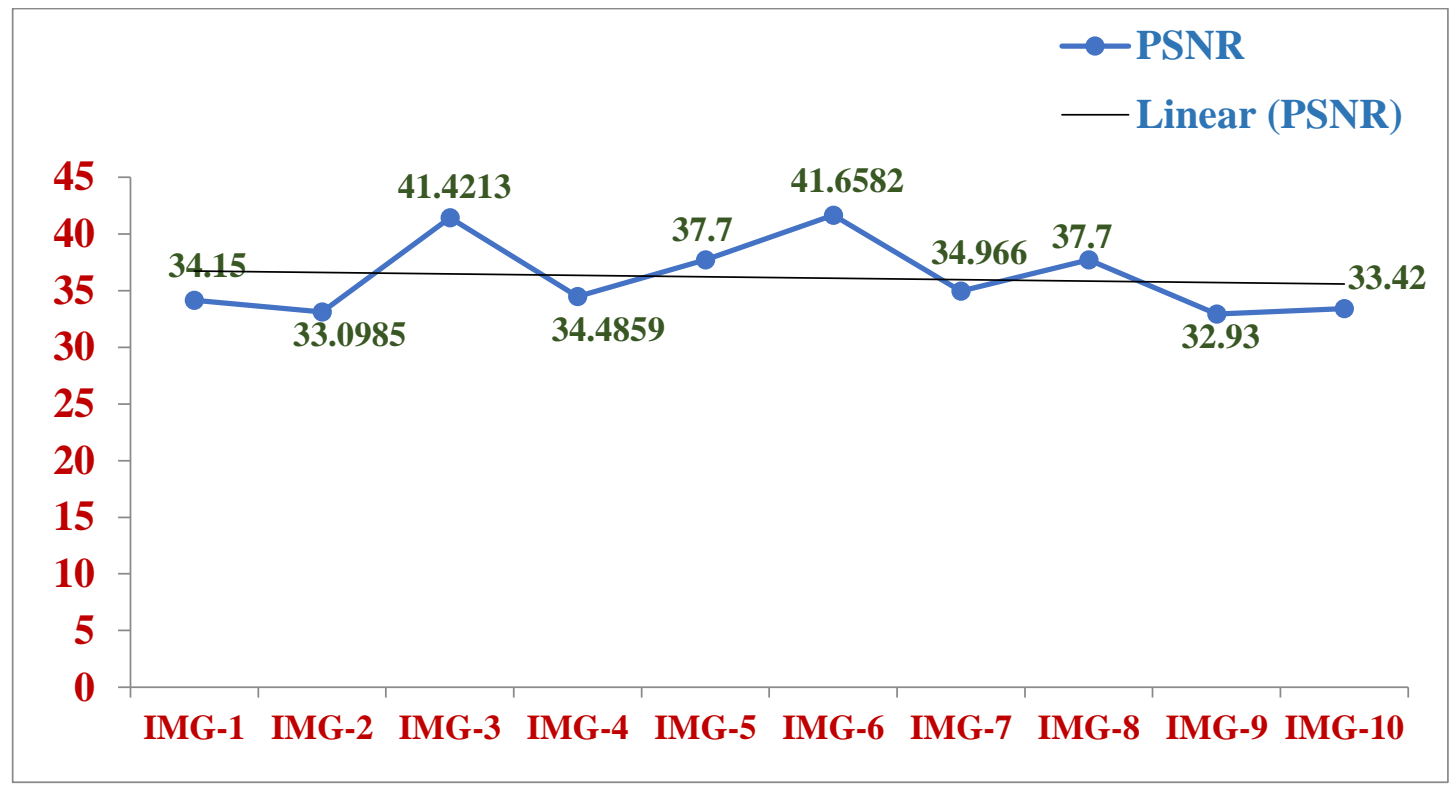

Fig.5 Analysis of PSNR for Various Brain Images using Hybrid Techniques 
Vol. 2 Iss. 1 Year 2020 Satyawati Magar and Bhavani Sridharan /2020

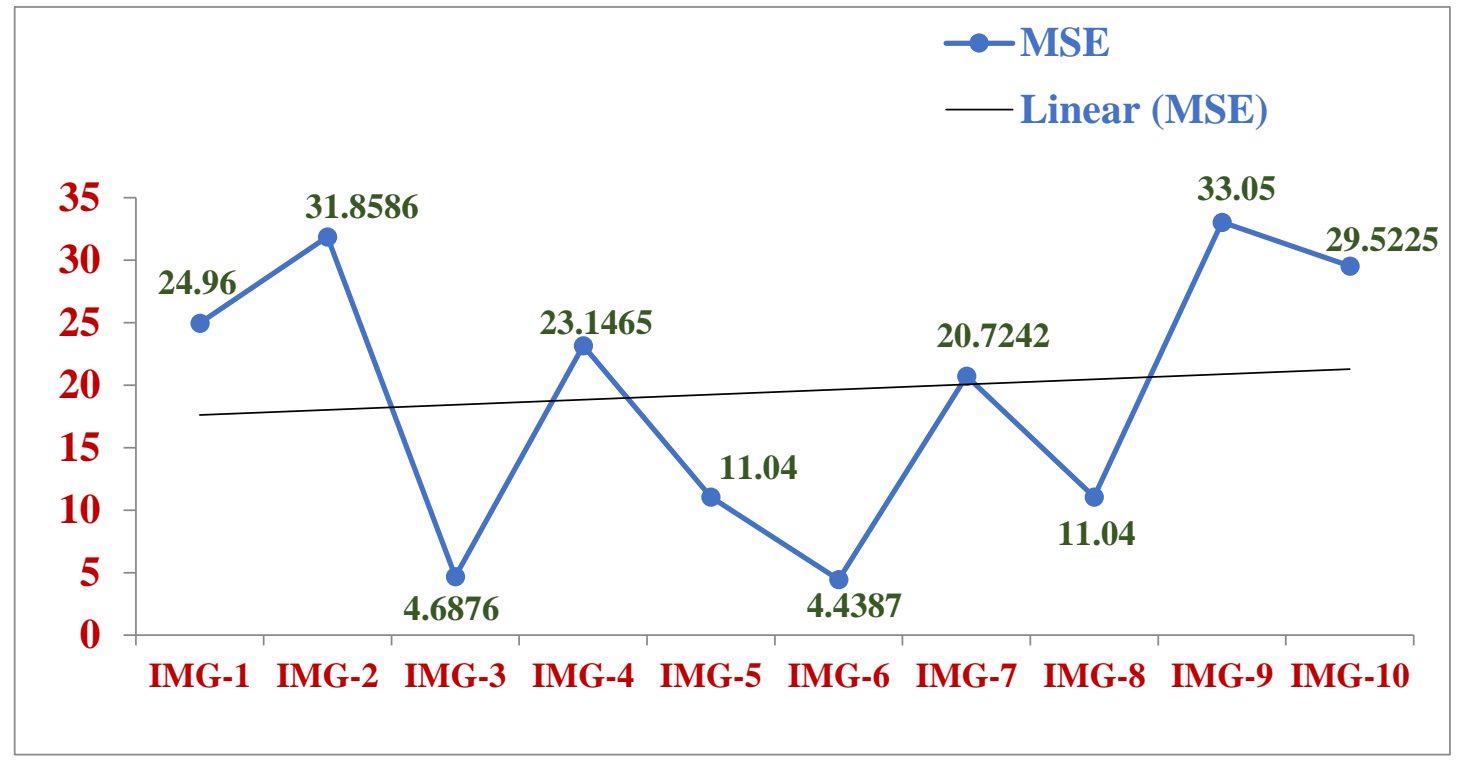

Fig.6 Analysis of MSE for Various Brain Images using Hybrid Techniques

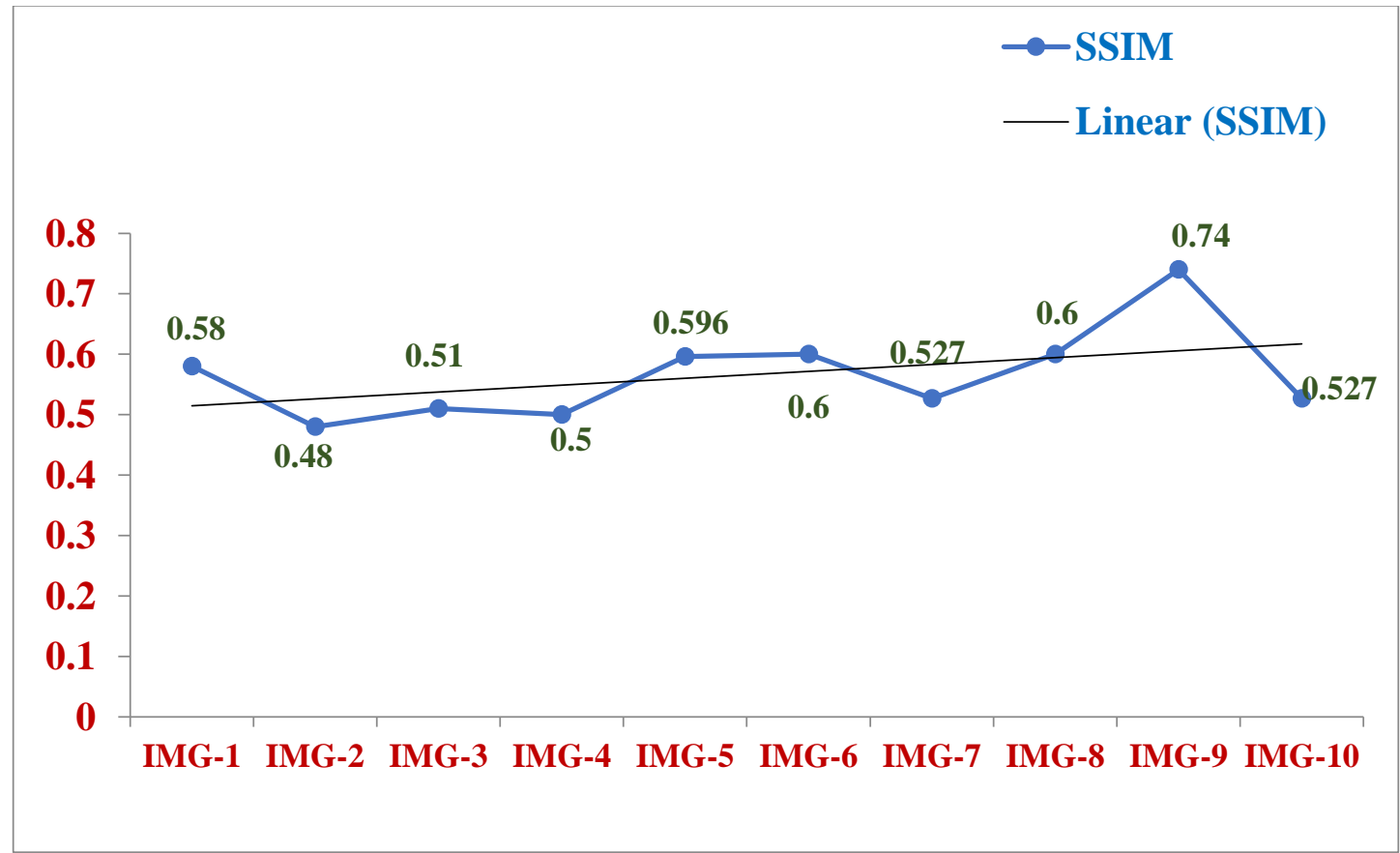

Fig.7 Analysis of SSIM for Various Brain Images using Hybrid Techniques 
Vol. 2 Iss. 1 Year $2020 \quad$ Satyawati Magar and Bhavani Sridharan /2020

Table 3 Average results of various Image Compression methods

\begin{tabular}{|l|l|l|l|l|l|}
\hline Sr. No. & Method /Parameters & CR & PSNR & MSE & SSIM \\
\hline $\mathbf{1}$ & $\begin{array}{l}\text { Hybrid (Oscillation \& } \\
\text { Quasi Fractal) }\end{array}$ & 22.956 & 36.153 & 19.449 & 0.566 \\
\hline $\mathbf{2}$ & Hybrid(BTC-SPIHT) & 5.492 & 35.248 & 33.43 & 0.547 \\
\hline
\end{tabular}

\section{Conclusion}

On comparing the results of both hybrid methods, it is observed that Innovative hybrid coding method using oscillation concept \& Quasi fractal gives better results than BTC-SPIHT hybrid method. CR is improved from 5.65 to 24.61 which is better. Also PSNR is retained \& little bit improved.

\section{References}

[1] Korakot Prachumrak, Akira Hiramatsu, Takayasu Fuchida, Hitofumi Nakamura and Sadayuki Murashima, Lossless Fractal Image Coding, 4th EURASIP Conference Focused on Video / Image Processing and Multimedia Communications. 2-5 July 2003.

[2] S. Bhavani \& Dr. K. Thanushkodi, A New Algorithm for Fractal Coding Using Self Organizing Map, Journal of Computer Science, 841- 845, 2012.

[3] Ju Cheng Yang, Dong Sun Park, Detecting Region-of-Interest (ROI) in Digital Mammogram by using Morphological Bandpass Filter, IEEE ,2004

[4] Francesco G.B. De Natale, Giulia Boato, Detecting Morphological Filtering of Binary Images, IEEE Transaction, 2016.

[5] Rahul Sharma, Chandrashekhar Kamargaonkar and Dr. Monisha Sharma, Hybrid Medical Image Compression: Survey, International Journal of Advanced Research in Computer Engineering \& Technology (IJARCET) Volume 5, Issue 4, April 2016.

[6] Alyaa H., Medical Image Data Compression Using Hybrid Methods, ARPN Journal of Engineering and Applied Sciences, VOL. 13, NO. 5, March 2018.

[7] Benamrane Nacera Bentorki Soumia, A Hybrid Scheme Coding Using SPIHT and Fractal for Mammography Image Compression, 15th International Conference on Information Visualization, IEEE Xplore, 2011.

[8] Nehal Markandeya, Sonali Patil, Digital Image Compression Hybrid Technique Based on Block Truncation Coding and Discrete Cosine Transform, International Conference on Trends in Electronics and Informatics, ICEI 2017. 
[9] Harjeetpal Singh, Sakshi Rana, Image Compression Hybrid using DCT, DWT, Huffman, International Journal of Scientific \& Engineering Research (IJSER), Volume 3, Issue 8, August-2012.

[10] Seddeq E. Ghrare \& Ahmed R. Khobaiz, Digital Image Compression Using Block Truncation Coding and Walsh Hadamard Transform Hybrid Technique, IEEE 2014 International Conference on Computer, Communication, and Control Technology (I4CT 2014), September 2-4, 2014.

[11] G. Soundarya, Comparison of Hybrid Codes for MRI Brain Image Compression, Research Journal of Applied Sciences, Engineering and Technology 4(24), 2012.

[12] Rafael C. Gonzalez, Richard E. Woods, Digital Image Processing, Pearson, Education, Inc., Second Edition, 2004.

[13] Rafael C. Gonzalez, Richard E. Woods, Steven Eddins, Digital image Processing using MATLAB', Pearson Education, Inc. ,2004.

[14] R.N. Chaudhary, Waves and Oscillations, New Edge International Publishers

\section{Acknowledgements}

The author declare that they have no conflict of interest.

\section{Conflict of interest}

The author declare that they have no conflict of interest.

\section{About The License}

(C) 2020 The Authors. This work is licensed under a Creative Commons Attribution 4.0 International License which permits unrestricted use, provided the original author and source are credited. 